\title{
Fast quantitative LC-MS/MS determination of illicit substances in solid and
}

liquid unknown seized samples

G.M. Merone ${ }^{1, \$}$, A. Tartaglia ${ }^{2, \$}$, S. Rossi ${ }^{~}$, F. Santavenere ${ }^{1}$, E. Bassotti ${ }^{3}$, C. D’Ovidio ${ }^{4}$, M. Bonelli $^{4}$, E. Rosato ${ }^{2}$, U. de Grazia ${ }^{5}$, M. Locatelli ${ }^{2, *}$, F. Savini ${ }^{1}$

1 Pharmatoxicology Laboratory - Hospital "Santo Spirito", Via Fonte Romana 8, Pescara 65124, Italy;

${ }^{2}$ Department of Pharmacy, University of Chieti-Pescara "G. d'Annunzio”, Via dei Vestini 31, Chieti 66100, Italy;

${ }^{3}$ R\&D Department Eureka Lab Division, Via Enrico Fermi, 25, Chiaravalle 60033, Italy;

${ }^{4}$ Department of Medicine and Aging Sciences, Section of Legal Medicine, University of ChietiPescara “G. d'Annunzio”, Chieti 66100, Italy;

${ }^{5}$ Fondazione IRCCS Istituto Neurologico Carlo Besta, Laboratory of Neurological Biochemistry and Neuropharmacology, Via Celoria 11, 20133 Milan, Italy.

${ }^{\$}$ These authors contributed equally

Corresponding author:

* Prof. Marcello Locatelli

Department of Pharmacy, University of Chieti-Pescara “G. d'Annunzio", Via dei Vestini 31, Chieti 66100, Italy;

https://orcid.org/0000-0002-0840-825X; E-mail: marcello.locatelli@unich.it; Phone: +3908713554590; Fax: +3908713554911. 


\section{Table of contents}

Table S.1 Detailed MRM transitions monitored in this method

Table S.2 Internal standards MRM transitions

Table S.3 Chromatographic elution profile

Figure S.1 Gradient curve

Table S.4 Mass spectrometer parameters

Figure S.2 MRM in positive (upper) and negative (lower) ionization mode for the calibrators C5

Table S.5 Analytical parameters of the method for the 37 analytes

Figures S.3 Some chromatograms related to the analyses of unknown seized materials performed on $19^{\text {th }}$ April 2021

Table S.6 Comparison with other methods in literature

Table S.7 General classification for GAPI index

Figure S.4 Correspondence to GAPI pictogram in the main text: 
Table S.1 Detailed MRM transitions monitored in this method

\begin{tabular}{|c|c|c|c|c|c|c|}
\hline Analyte & $\begin{array}{c}\text { Internal Standard used as } \\
\text { reference }\end{array}$ & Q1 (m/z) & $\begin{array}{c}\text { Q3 }(\mathrm{m} / \mathrm{z}) \\
\text { Quantifier }\end{array}$ & $\begin{array}{l}\text { Q3 }(\mathrm{m} / \mathrm{z}) \\
\text { Qualifier }\end{array}$ & Retention time (min) & Polarity \\
\hline Cocaine & Cocaine D3 & 304.2 & 182.2 & 82.2 & 5.5 & + \\
\hline 6-MAM & 6-MAM D6 & 328.1 & 165.2 & 211.1 & 3.4 & + \\
\hline Amphetamine & Morphine D6 & 136.1 & 91.1 & 119.2 & 2.2 & + \\
\hline Methamphetamine & Morphine D6 & 150.2 & 91.1 & 119.2 & 3.2 & + \\
\hline MDA & Morphine D6 & 180.1 & 133.1 & 135.1 & 3.0 & + \\
\hline MDE & Morphine D6 & 208.2 & 163.1 & 135.1 & 4.5 & + \\
\hline MDMA & Morphine D6 & 194.1 & 163.1 & 105.1 & 3.7 & + \\
\hline MBDB & Morphine D6 & 208.2 & 135.2 & 177.1 & 4.6 & + \\
\hline Ketamine & Morphine D6 & 238.2 & 125.0 & 179.1 & 4.8 & + \\
\hline Methorphan & Cocaine D3 & 272.2 & 171.2 & 215.2 & 6.9 & + \\
\hline Buprenorphine & Buprenorphine D4 & 468.3 & 396.2 & 414.3 & 7.2 & + \\
\hline Methadone & Methadone D9 & 310.2 & 265.2 & 105.2 & 8.4 & + \\
\hline Morphine & Morphine D3 & 286.1 & 152.1 & 165.1 & 1.1 & + \\
\hline Aminophenazone & 6-MAM D6 & 232.1 & 113.1 & 97.1 & 2.5 & + \\
\hline Caffeine & 6-MAM D6 & 195.1 & 138.1 & 110.0 & 3.4 & + \\
\hline Diltiazem & Cocaine D3 & 415.2 & 178.2 & 150.1 & 7.2 & + \\
\hline Phenacetin & Cocaine D3 & 180.1 & 110.1 & 138.2 & 4.4 & + \\
\hline Hydroxyzine & Cocaine D3 & 375.2 & 201.1 & 166.1 & 7.8 & + \\
\hline Lidocaine & Cocaine D3 & 235.2 & 86.1 & 58.0 & 4.3 & + \\
\hline Naloxone & 6-MAM D6 & 328.2 & 310.2 & 212.1 & 3.1 & + \\
\hline Noscapine & Cocaine D3 & 414.2 & 220.2 & 353.2 & 6.3 & + \\
\hline Paracetamol & Morphine D6 & 152.1 & 110.1 & 65.0 & 1.4 & + \\
\hline $\mathrm{CBD}$ & THC D3 & 315.3 & 193.1 & 123.1 & 8.4 & + \\
\hline Acetylsalicylic acid & 6-MAM D6 & 179.0 & 137.0 & 93.0 & 10 & - \\
\hline Paroxetine & Methadone D9 & 330.2 & 69.9 & 192.2 & 7.8 & + \\
\hline Procaine & Cocaine D3 & 237.2 & 100.1 & 120.0 & 3.2 & + \\
\hline Procainamide & Cocaine D3 & 236.2 & 163.2 & 120.1 & 1.7 & + \\
\hline Sulfametoxazole & 6-MAM D6 & 254.1 & 92.1 & 108.1 & 4.0 & + \\
\hline
\end{tabular}


Table S.1 cont. Detailed MRM transitions monitored in this method

\begin{tabular}{|c|c|c|c|c|c|c|}
\hline Analyte & $\begin{array}{c}\text { Internal Standard used as } \\
\text { reference }\end{array}$ & Q1 (m/z) & $\begin{array}{c}\text { Q3 }(\mathbf{m} / \mathbf{z}) \\
\text { Quantifier }\end{array}$ & $\begin{array}{l}\text { Q3 (m/z) } \\
\text { Qualifier }\end{array}$ & Retention time (min) & Polarity \\
\hline Trimethoprim & 6-MAM D6 & 291.1 & 230.2 & 261.2 & 3.6 & + \\
\hline Diacetylmorphine & Morphine D6 & 370.2 & 165.0 & 268.1 & 5.1 & + \\
\hline Levamisole & 6-MAM D6 & 205.1 & 177.8 & 122.8 & 3.7 & + \\
\hline Tropacocaine & Cocaine D3 & 246.4 & 123.9 & 95.6 & 5.2 & + \\
\hline Benzocaine & Cocaine D3 & 166.1 & 138.0 & 77.0 & 4.6 & + \\
\hline Nicotine & Morphine D6 & 163.1 & 130.1 & 117.1 & 0.7 & + \\
\hline Ephedrine & 6-MAM D6 & 166.1 & 148.4 & 91.1 & 2.1 & + \\
\hline Pseudoephedrine & 6-MAM D6 & 166.1 & 148.0 & 91.1 & 2.7 & + \\
\hline
\end{tabular}

Table S.2 Internal standards MRM transitions

\begin{tabular}{ccccc}
\hline Analyte & Q1 $(\mathbf{m} / \mathbf{z})$ & $\mathbf{Q 3}(\mathbf{m} / \mathbf{z})$ & Retention time (min) & Polarity \\
\hline Cocaine D3 & 307.2 & 185.2 & 5.5 & + \\
THC D3 & 318.2 & 196.2 & 9.1 & + \\
Methadone D9 & 319.2 & 268.2 & 8.4 & + \\
Morphine D6 & 292.1 & 181.1 & 1.1 & + \\
Buprenorphine D4 & 472.3 & 400.2 & 7.2 & + \\
6MAM D6 & 334.1 & 211.1 & 3.4 & + \\
\hline
\end{tabular}


Table S.3 Chromatographic elution profile

\begin{tabular}{cccc}
\hline Time $(\mathrm{min})$ & Flow rate $(\mathrm{mL} / \mathrm{min})$ & Mobile phase A & Mobile phase B \\
\hline 0 & & 95 & 5 \\
0.2 & & 95 & 5 \\
8 & & 25 & 75 \\
8.1 & 0.4 & 0 & 100 \\
10 & & 0 & 100 \\
10.1 & & 95 & 5 \\
15 & & 95 & 5 \\
\hline
\end{tabular}

Mobile phase $\mathrm{M} 1: \mathrm{H}_{2} \mathrm{O}, 0.1 \%$ formic acid, $10 \mathrm{mM}$ ammonium formate; Mobile phase M2: acetonitrile

Column: Hypersil Gold PFP $(50 \times 2.1 \mathrm{~mm}, 1.9 \mu \mathrm{m})$ thermostated at $40^{\circ} \mathrm{C}$

Figure S.1 Gradient curve

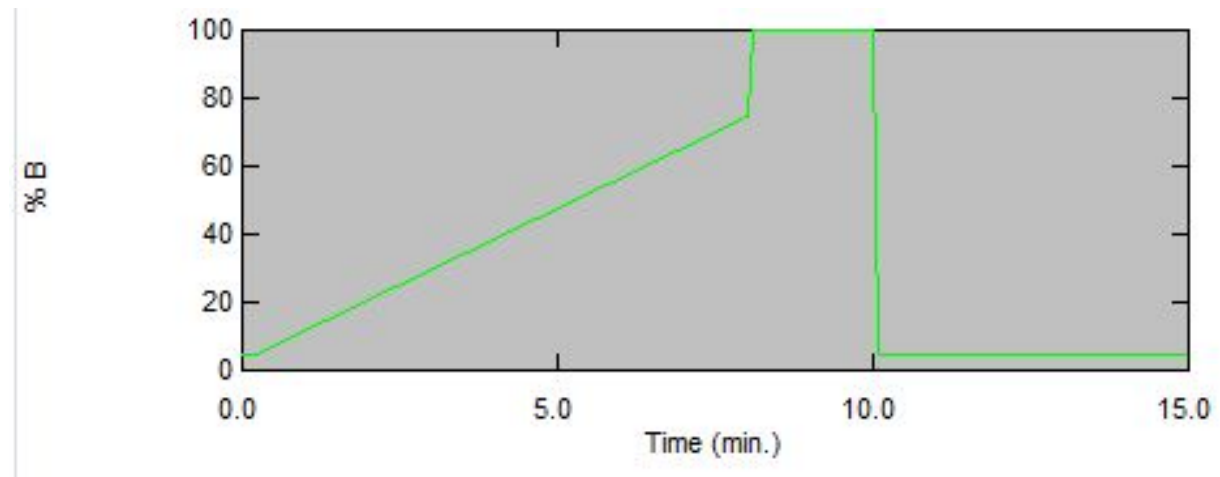

Table S.4 Mass spectrometer parameters

\begin{tabular}{cll}
\hline Ionization mode & \multicolumn{1}{c}{ Parameter } & \multicolumn{1}{c}{ Value } \\
& Curtain gas (CUR) & 25 \\
& Collision Gas (CAD) & Medium \\
& Ion Spray Voltage (IS) & 5400 \\
POSITIVE & Temperature (TEM) & $450^{\circ} \mathrm{C}$ \\
& Ion Source Gas 1 (GS1) & 55 \\
& Ion Source Gas 2 (GS2) & 55 \\
\hline \hline \multirow{5}{*}{ NEGATIVE } & Curtain gas (CUR) & 25 \\
& Collision Gas (CAD) & Medium \\
& Ion Spray Voltage (IS) & -4500 \\
& Temperature (TEM) & $450^{\circ} \mathrm{C}$ \\
& Ion Source Gas 1 (GS1) & 55 \\
& Ion Source Gas 2 (GS2) & 55 \\
\hline
\end{tabular}


Figure S.2 MRM in positive (upper) and negative (lower) ionization mode for the calibrators C5

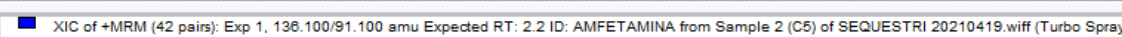
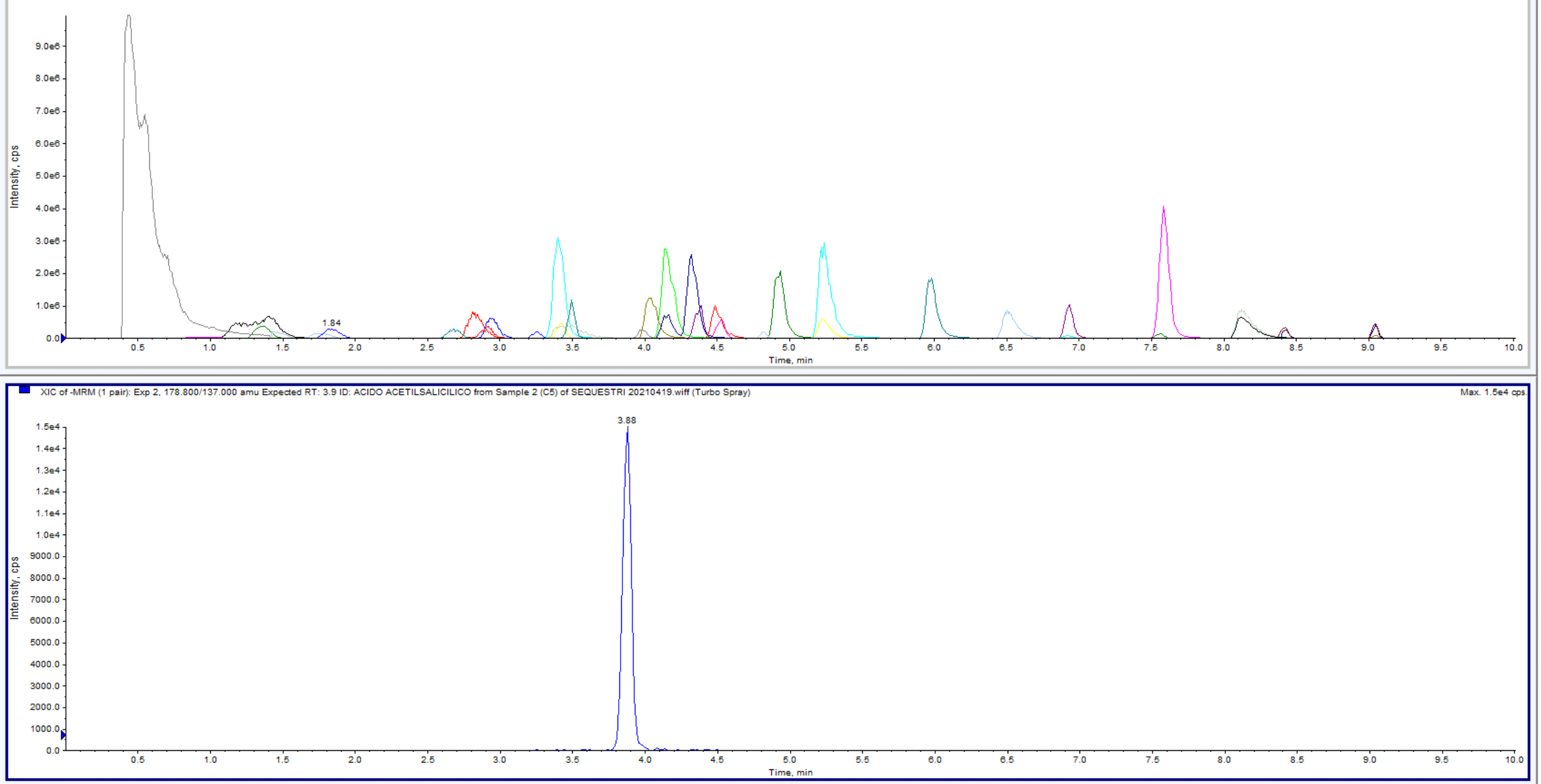
Table S.5 Analytical parameters of the method for the 37 analytes

\begin{tabular}{|c|c|c|c|c|c|c|c|c|c|c|c|c|c|c|}
\hline \multirow{3}{*}{ Parameter } & \multirow{3}{*}{$\begin{array}{l}\text { Range } \\
(\mathrm{ng} / \mathrm{mL})\end{array}$} & \multirow{3}{*}{$\begin{array}{c}\text { LLOD } \\
(\mathrm{ng} / \mathrm{mL})\end{array}$} & \multirow{3}{*}{$\begin{array}{c}\text { LLOQ } \\
(\mathrm{ng} / \mathrm{mL})\end{array}$} & \multirow{3}{*}{$\begin{array}{l}\text { Correlation } \\
\text { Coeff. }\left(r^{2}\right)^{\mathrm{a}}\end{array}$} & \multicolumn{6}{|c|}{ Precision (CV\%) } & \multicolumn{4}{|c|}{ Trueness (BIAS\%) } \\
\hline & & & & & \multicolumn{3}{|c|}{ Intraday } & \multicolumn{3}{|c|}{ Interday } & \multicolumn{2}{|c|}{ Intraday } & \multicolumn{2}{|c|}{ Interday } \\
\hline & & & & & LLOQ & $\mathbf{C}_{\mathrm{m}}$ & $\mathbf{C}_{\mathrm{up}}$ & LLOQ & $\mathbf{C}_{\mathbf{m}}$ & $\mathbf{C}_{\text {up }}$ & $\mathbf{C}_{\mathbf{i}}$ & $\mathrm{C}_{\mathrm{h}}$ & $\mathbf{C}_{\mathbf{i}}$ & $\mathbf{C}_{\mathrm{h}}$ \\
\hline Cocaine & & & & $0.9945 \pm 0.0014$ & 2.63 & 5.59 & 3.98 & 4.13 & 9.04 & 8.86 & 6.47 & 3.11 & 6.23 & 2.54 \\
\hline 6-MAM & & & & $0.9942 \pm 0.0027$ & 2.99 & 1.90 & 3.97 & 7.01 & 4.68 & 9.28 & 5.50 & 1.32 & 6.11 & 2.87 \\
\hline Amphetamine & & & & $0.9923 \pm 0.0012$ & 3.55 & 4.87 & 2.08 & 9.91 & 9.88 & 8.60 & 7.46 & 5.16 & 8.88 & 6.01 \\
\hline Methamphetamine & & & & $0.9921 \pm 0.0021$ & 6.57 & 7.84 & 3.66 & 8.67 & 9.12 & 7.57 & 10.8 & 1.77 & 11.0 & 1.90 \\
\hline MDA & & & & $0.9914 \pm 0.0017$ & 1.31 & 2.88 & 5.36 & 7.75 & 6.30 & 8.95 & 6.58 & 2.71 & 7.59 & 3.58 \\
\hline MDE & & & & $0.9939 \pm 0.0014$ & 6.74 & 5.84 & 5.36 & 10.7 & 6.78 & 8.72 & 6.76 & 2.70 & 10.9 & 3.36 \\
\hline MDMA & & & & $0.9945 \pm 0.0023$ & 7.91 & 6.52 & 3.80 & 9.57 & 10.5 & 7.50 & 4.97 & 3.10 & 8.55 & 4.38 \\
\hline MBDB & & & & $0.9935 \pm 0.0019$ & 2.80 & 6.97 & 3.33 & 9.15 & 8.08 & 6.70 & 6.94 & 2.82 & 9.73 & 3.77 \\
\hline Ketamine & & & & $0.9928 \pm 0.0018$ & 4.25 & 7.28 & 4.79 & 9.41 & 8.32 & 8.33 & 6.69 & 2.56 & 9.73 & 3.96 \\
\hline Methorphan & $5-100$ & 1.67 & 5 & $0.9946 \pm 0.0030$ & 3.43 & 5.10 & 3.77 & 7.10 & 5.65 & 8.65 & 6.91 & 1.90 & 7.67 & 4.39 \\
\hline Buprenorphine & & & & $0.9929 \pm 0.0011$ & 4.54 & 4.01 & 2.11 & 5.78 & 8.03 & 6.65 & 5.91 & 0.35 & 7.08 & 3.12 \\
\hline Methadone & & & & $0.9966 \pm 0.0011$ & 0.89 & 1.65 & 1.65 & 7.39 & 7.18 & 3.68 & 6.28 & 1.74 & 9.30 & 3.25 \\
\hline Morphine & & & & $0.9950 \pm 0.0019$ & 3.91 & 4.94 & 0.85 & 7.49 & 7.95 & 8.12 & 4.88 & 2.16 & 8.26 & 2.79 \\
\hline THC & & & & $0.9952 \pm 0.0024$ & 4.64 & 4.14 & 4.75 & 6.19 & 8.36 & 8.73 & 6.89 & 6.03 & 8.51 & 4.75 \\
\hline Aminophenazone & & & & $0.9929 \pm 0.0029$ & 3.89 & 5.52 & 6.66 & 12.5 & 11.5 & 13.4 & 2.27 & 1.65 & 5.21 & 2.41 \\
\hline Caffeine & & & & $0.9919 \pm 0.0029$ & 4.54 & 5.67 & 5.59 & 8.27 & 6.94 & 7.38 & 4.13 & 2.34 & 5.81 & 3.82 \\
\hline Diltiazem & & & & $0.9936 \pm 0.0021$ & 1.50 & 5.05 & 3.49 & 5.52 & 8.69 & 7.89 & 4.40 & 2.16 & 9.15 & 2.55 \\
\hline Phenacetin & & & & $0.9943 \pm 0.0017$ & 2.87 & 2.16 & 3.98 & 7.42 & 10.6 & 10.9 & 4.48 & 3.27 & 6.70 & 3.88 \\
\hline Hydroxyzine & & & & $0.9959 \pm 0.0028$ & 2.08 & 1.59 & 1.53 & 6.57 & 5.98 & 3.69 & 13.4 & 7.10 & 11.2 & 9.88 \\
\hline
\end{tabular}


Table S.5 cont. Analytical parameters of the method for the 37 analytes

\begin{tabular}{|c|c|c|c|c|c|c|c|c|c|c|c|c|c|c|}
\hline \multirow{3}{*}{ Parameter } & \multirow{3}{*}{$\begin{array}{c}\text { Range } \\
\text { (ng/mL) }\end{array}$} & \multirow{3}{*}{$\begin{array}{c}\text { LLOD } \\
(\mathrm{ng} / \mathrm{mL})\end{array}$} & \multirow{3}{*}{$\begin{array}{c}\text { LLOQ } \\
\text { (ng/mL) }\end{array}$} & \multirow{3}{*}{$\begin{array}{l}\text { Correlation } \\
\text { Coeff. }\left(r^{2}\right)^{a}\end{array}$} & \multicolumn{6}{|c|}{ Precision (CV\%) } & \multicolumn{4}{|c|}{ Trueness (BIAS\%) } \\
\hline & & & & & \multicolumn{3}{|c|}{ Intraday } & \multicolumn{3}{|c|}{ Interday } & \multicolumn{2}{|c|}{ Intraday } & \multicolumn{2}{|c|}{ Interday } \\
\hline & & & & & LLOQ & $\mathbf{C}_{\mathbf{m}}$ & $\mathbf{C}_{\text {up }}$ & LLOQ & $\mathbf{C}_{\mathbf{m}}$ & $\mathbf{C}_{\mathrm{up}}$ & $\mathbf{C}_{\mathbf{i}}$ & $\mathbf{C}_{\mathrm{h}}$ & $\mathbf{C}_{\mathbf{i}}$ & $\mathbf{C}_{\mathrm{h}}$ \\
\hline Naloxone & \multirow{12}{*}{$5-100$} & \multirow{12}{*}{1.67} & \multirow{12}{*}{5} & $0.9923 \pm 0.0023$ & 4.69 & 5.23 & 6.08 & 12.8 & 9.89 & 11.4 & 2.83 & 3.50 & 6.83 & 4.41 \\
\hline Noscapine & & & & $0.9953 \pm 0.0033$ & 4.01 & 7.14 & 5.27 & 5.46 & 8.60 & 9.73 & 1.31 & 1.75 & 4.51 & 2.70 \\
\hline Acetylsalicylic acid & & & & $0.9978 \pm 0.0009$ & 3.60 & 5.40 & 1.20 & 4.90 & 7.20 & 5.30 & 3.60 & 5.00 & 8.20 & 7.20 \\
\hline Paroxetine & & & & $0.9917 \pm 0.0068$ & 3.73 & 3.79 & 4.97 & 5.71 & 7.39 & 7.55 & 7.08 & 3.19 & 8.82 & 6.08 \\
\hline Procaine & & & & $0.9922 \pm 0.0008$ & 5.48 & 5.13 & 2.85 & 6.19 & 8.87 & 8.91 & 6.21 & 6.77 & 8.54 & 9.78 \\
\hline Procainamide & & & & $0.9929 \pm 0.0015$ & 2.27 & 4.75 & 3.39 & 9.80 & 8.72 & 9.81 & 9.23 & 6.55 & 13.4 & 8.36 \\
\hline Levamisole & & & & $0.9921 \pm 0.0035$ & 3.31 & 5.79 & 4.71 & 9.47 & 10.8 & 9.86 & 10.1 & 4.70 & 10.3 & 9.03 \\
\hline Tropococaine & & & & $0.9915 \pm 0.0055$ & 4.16 & 5.94 & 2.65 & 4.52 & 7.49 & 9.34 & 5.65 & 2.29 & 7.35 & 4.19 \\
\hline Benzocaine & & & & $0.9950 \pm 0.0043$ & 4.89 & 7.74 & 3.46 & 7.70 & 8.07 & 9.46 & 3.78 & 2.20 & 6.85 & 3.95 \\
\hline Nicotine & & & & $0.9945 \pm 0.0011$ & 3.94 & 7.46 & 5.89 & 4.16 & 6.47 & 6.27 & 8.20 & 2.30 & 9.15 & 5.60 \\
\hline Ephedrine, & & & & $0.9911 \pm 0.0064$ & 5.78 & 7.80 & 6.77 & 14.3 & 7.84 & 9.25 & 2.92 & 1.27 & 7.53 & 9.16 \\
\hline Pseudoephedrine & & & & $0.9911 \pm 0.0064$ & 6.60 & 8.10 & 7.30 & 10.5 & 9.60 & 10.2 & 3.50 & 3.00 & 8.20 & 8.60 \\
\hline
\end{tabular}

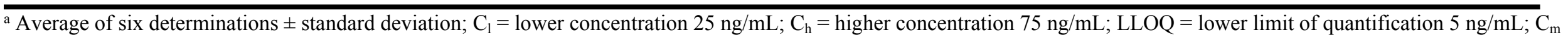

= medium concentration $50 \mathrm{ng} / \mathrm{mL} ; \mathrm{C}_{\mathrm{up}}=$ upper concentration $100 \mathrm{ng} / \mathrm{mL}$; LLOD = lower limit of detection. 
Figures S.3 Some chromatograms related to the analyses of unknown seized materials performed on $19^{\text {th }}$ April 2021

Transitions used for the identification of unknown seized material:

Cocaine $\quad \rightarrow \quad$ Cocaine + Cocaine D3

Marijuana/hashish $\rightarrow$ CBD, THC + THC D3

Heroin $\quad \rightarrow \quad 6 \mathrm{MAM}+6 \mathrm{MAM}$ D6, caffeine, morphine, noscapine, paracetamol

Identified material: COCAINE

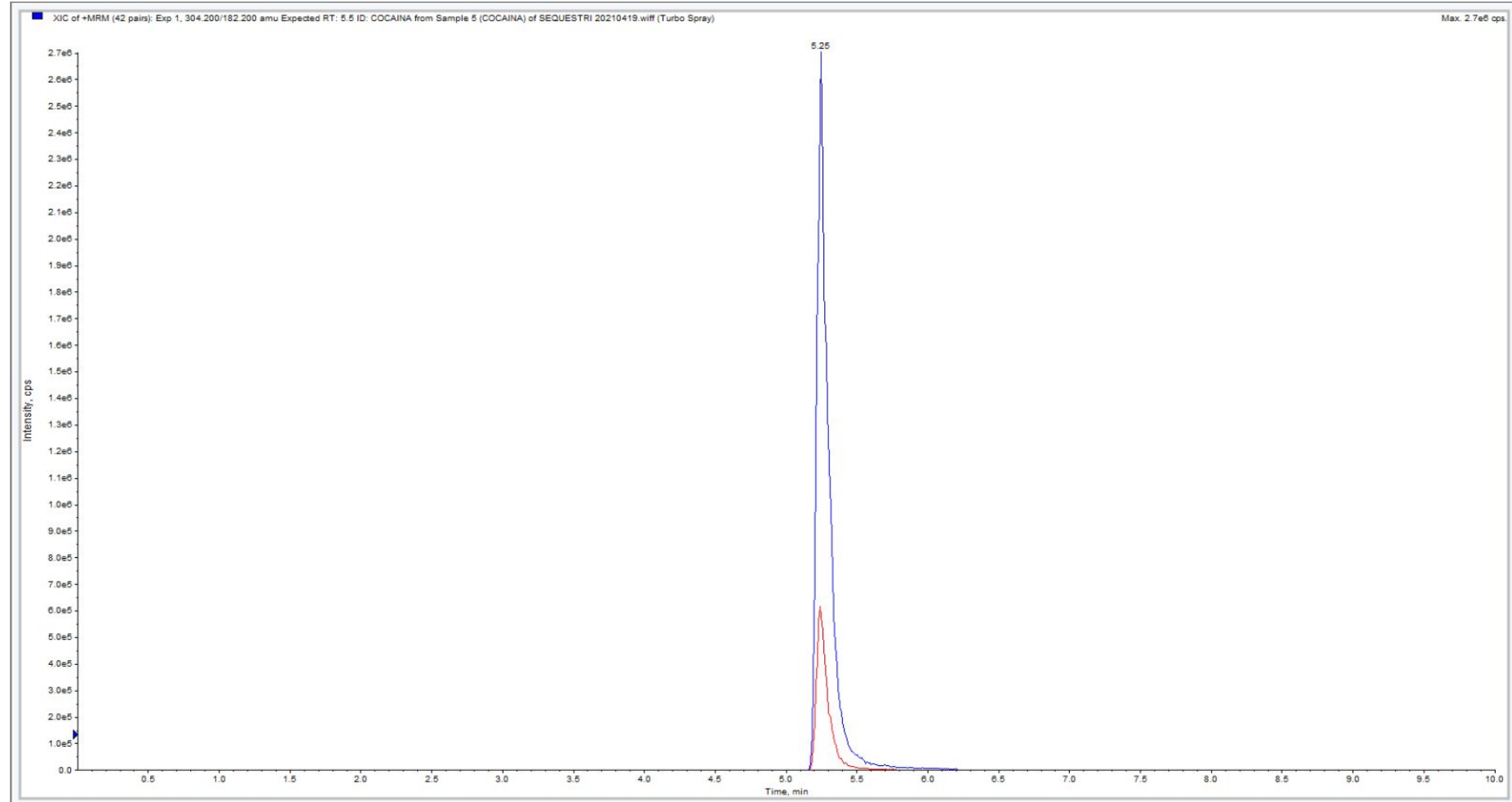




\section{Identified material: HASHISH}

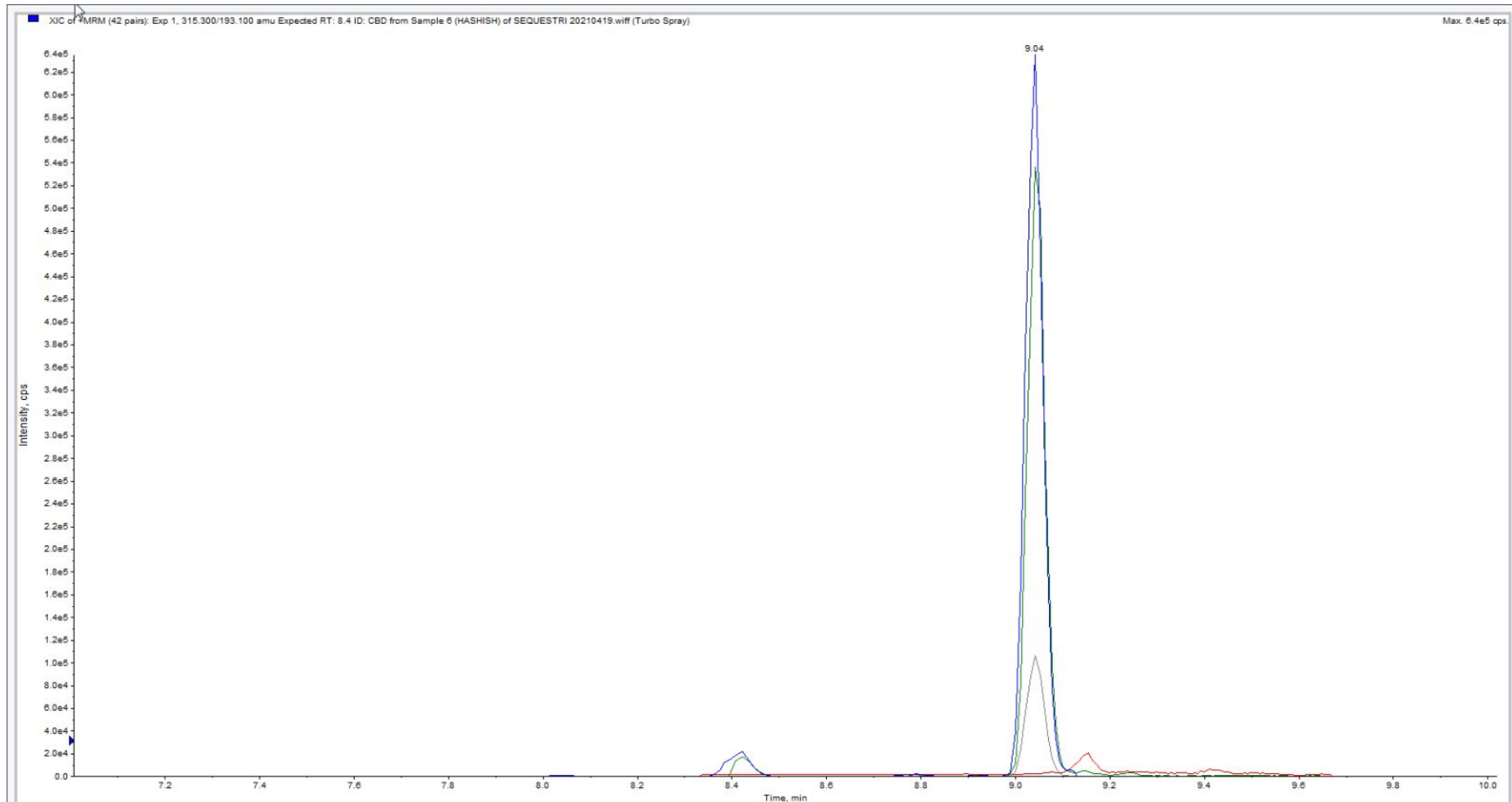

Identified material: MARIJUANA

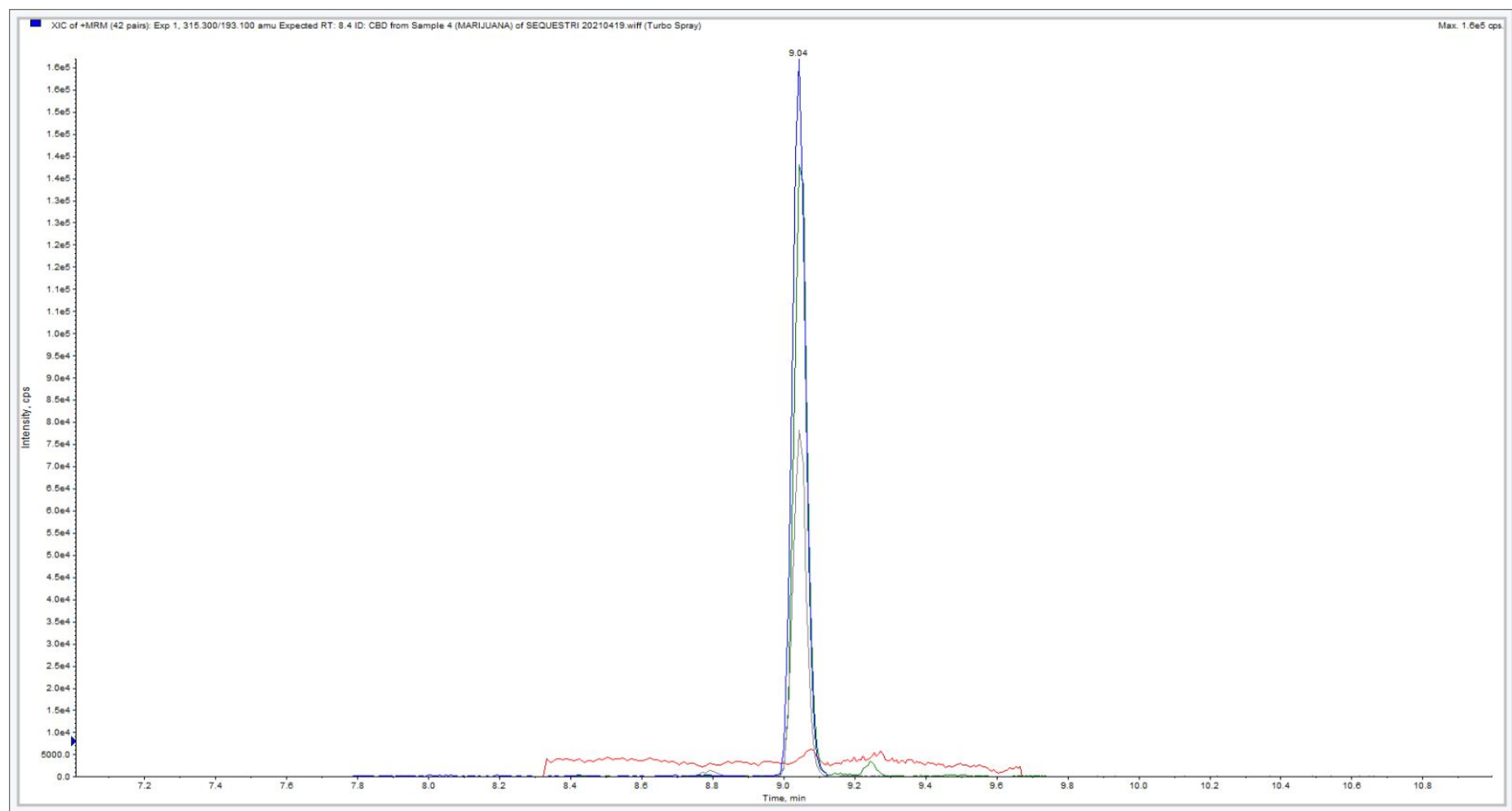


Identified material: HEROIN

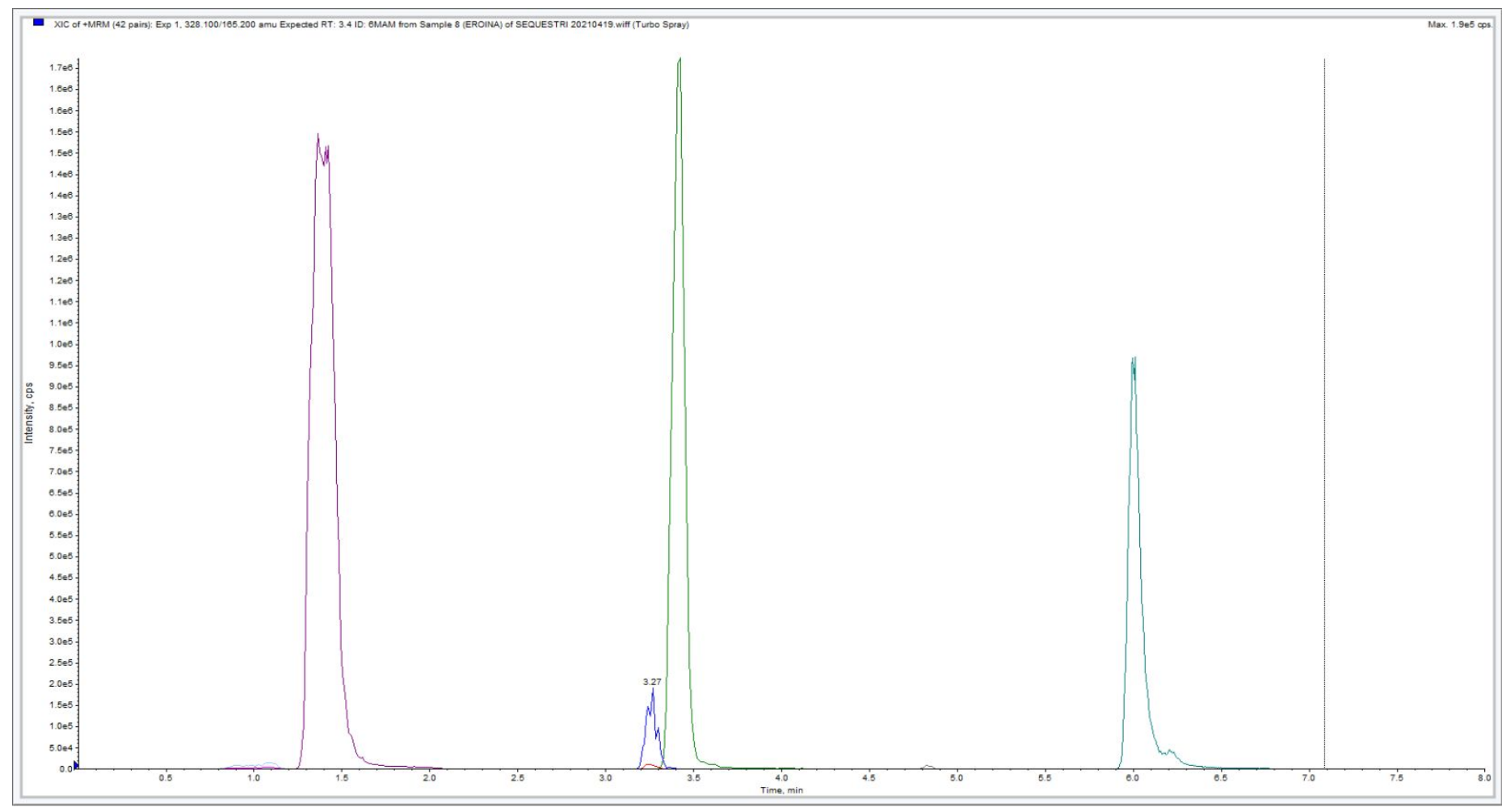

Identified material: 3,4-METHYLENEDIOXYMETHAMPHETAMINE or MDMA

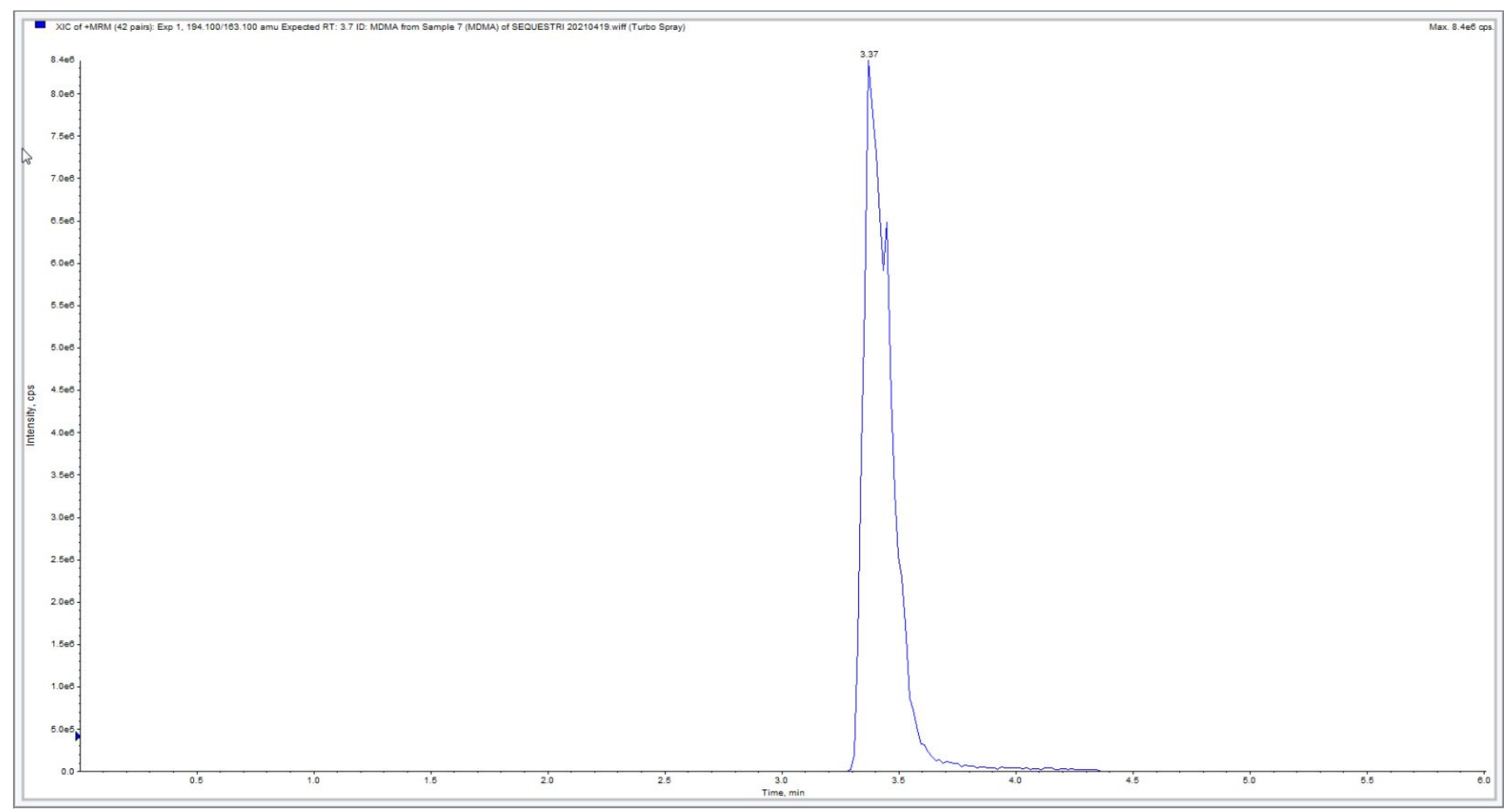


Table S.6 Comparison with other methods in literature

\begin{tabular}{|c|c|c|c|c|c|c|}
\hline Analytes & Matrix & Treatment & Instrumentation & Runtime & LOD/LOQ & Ref. \\
\hline Methamphetamine & Seized street samples & - & $\begin{array}{l}\text { Surface-enhanced } \\
\text { Raman sensing }\end{array}$ & - & LOD $7 \mathrm{pM}$ & $(1)$ \\
\hline Cocaine, heroin, THC & Solid seized material & Dilution & $\begin{array}{l}\text { GC-MS (qualitative) } \\
\text { GC-FID (quantitative) }\end{array}$ & $\begin{array}{l}20 \mathrm{~min} \\
12 \mathrm{~min}\end{array}$ & $\begin{array}{l}\text { LOD } 3 \mathrm{ng} / \mu \mathrm{L} \\
\text { LLOQ } 4.2 \mathrm{ng} / \mu \mathrm{L}\end{array}$ & $(2)$ \\
\hline $\begin{array}{l}\mathrm{Cl}^{-}, \mathrm{NO}_{2}^{-}, \mathrm{NO}_{3}^{-}, \mathrm{SO}_{4}^{2-}, \mathrm{CO}_{3}^{2-}, \mathrm{BO}_{3}^{3-}, \text { Levamisole, } \\
\text { Lidocaine, Cocaine }\end{array}$ & Solid seized material & Dilution & CE-UV & $3 \mathrm{~min}$ & $\begin{array}{l}\text { LOD } 1.5-3 \times 10^{-2} \mathrm{mmol} / \mathrm{L} \\
\text { LOQ } 0.05-1.2 \mathrm{mmol} / \mathrm{L}\end{array}$ & (3) \\
\hline $\begin{array}{l}\text { Cathinones, synthetic cannabinoids, miscellanea } \\
\text { ( } 32 \text { compounds) }\end{array}$ & Solid seized material & Dilution & $\begin{array}{l}\text { GC-MS } \\
\text { LC-HRMS }\end{array}$ & $\begin{array}{l}17.8 \mathrm{~min} \\
18.5 \mathrm{~min}\end{array}$ & $\begin{array}{l}\text { Qualitative analyses, no } \\
\text { LOD and LOQ }\end{array}$ & (4) \\
\hline $\begin{array}{l}\text { Alprazolam, amphetamine, aminopyrine, } \\
\text { benzocaine, caffeine, cocaine, codeine, diltiazem, } \\
\text { ephedrine, fentanyl, fenethylline, furanylfentanyl, } \\
\text { heroin, hydroxyzine, levamisole, lidocaine, } \\
\text { methamphetamine, morphine, noramidopyrine, } \\
\text { phencyclidine, phenacetin, procaine, strychnine, } \\
\text { xylazine }\end{array}$ & Solid seized material & Dilution & Portable GC-MS & $15 \min$ & $\begin{array}{l}\text { Qualitative analyses, LOD } \\
\text { from } 0.01 \text { to } 0.1 \mathrm{mg} / \mathrm{mL} \text {, no } \\
\text { LOQ }\end{array}$ & $(5)$ \\
\hline Amphetamine & Solid seized material & $\begin{array}{l}\text { Dilution } \\
\text { derivatization }\end{array}$ & Portable SWV & - & $\begin{array}{l}\text { LOD } 22.2 \mu \mathrm{M} \\
\text { LOQ } 50 \mu \mathrm{M}\end{array}$ & $(6)$ \\
\hline 54 different fentanyl analogues & Standard solution & - & GC-MS & $16 \min$ & PCA cluster classification & (7) \\
\hline Noscapine, heroin, papaverine, acetyl thebaol & Seized heroin samples & - & LC-MS/MS & $6 \mathrm{~min}$ & $\begin{array}{l}\text { LOD } 0.4 \mathrm{mg} / \mathrm{L} \\
\text { LOQ } 1.0 \mathrm{mg} / \mathrm{L}\end{array}$ & (8) \\
\hline
\end{tabular}


Table S.6 cont. Comparison with other methods in literature

\begin{tabular}{|c|c|c|c|c|c|c|}
\hline Analytes & Matrix & Treatment & Instrumentation & Time & LOD/LOQ & Ref. \\
\hline Fentanyl & Seized drug samples & - & LFIA & - & Qualitative analyses & (9) \\
\hline Ketamine & $\begin{array}{l}\text { Simulated street } \\
\text { samples }\end{array}$ & Dilution & $\begin{array}{l}\text { Portable potentiostat } \\
\text { (voltammetry) }\end{array}$ & - & Qualitative analyses & $(10)$ \\
\hline $\begin{array}{l}\text { Methamphetamine, ketamine, cocaine, } \\
\text { and cutting agents }\end{array}$ & Seized drug samples & Solid analysis & Raman spectroscopy & - & $\begin{array}{l}\text { Qualitative analyses, LOD from } \\
5 \% \text { to } 20 \% \text { of the material }\end{array}$ & (11) \\
\hline MDMA & Seized ecstasy tablets & Dilution & LC-DAD & $15 \mathrm{~min}$ & $\begin{array}{l}\text { LOD } 0.7 \mu \mathrm{g} / \mathrm{mL} \\
\text { LOQ } 3.1 \mu \mathrm{g} / \mathrm{mL}\end{array}$ & (12) \\
\hline
\end{tabular}

Cocaine, buprenorphine, amphetamine, methamphetamine, MDMA, MDA,

MDE, MBDB, ketamine,

diacetylmorphine, ephedrine,

pseudoephedrine, methadone,

methorphan, 6-MAM, THC, morphine,

acetylsalicylic acid, aminophenazone,

benzocaine, caffeine, diltiazem,

Seized solid and liquid

samples
Dilution

LC-MS/MS
LOD $1.67 \mathrm{ng} / \mathrm{mL}$

LOQ $5 \mathrm{ng} / \mathrm{mL}$
Current

validated

method

phenacetin, ibuprofen, hydroxyzine,

levamisole, lidocaine, naloxone,

nicotine, noscapine, paracetamol,

paroxetine, procaine, procainamide,

trimetoprim, sulfametoxazole,

tropacocaine

PP: protein precipitation; LC-MS/MS: liquid chromatography-tandem mass spectrometry; GC-MS: gas chromatography-mass spectrometry; GC-FID: gas chromatography-flame ionization detector; CE-UV: capillary electrophoresis-spectrophotometric detector; LC-HRMS: liquid chromatography-high resolution mass spectrometry; SWV: square wave

voltammetry sensor; LFIA: later flow immunoassay;

\section{References}

(1) Mao, J.; Kang, Y.; Yu, D.; Zhou, J. Anal. Chim. Acta 2021, 1146, 124-130.

(2) Silvestre, A.; Basilicata, P.; Coraggio, L.; Guadagni, R.; Si-monelli, A.; Pieri, M. Forensic Sci. Int. 2021, $321,110738$. 
(3) Barreto, D.N.; Ribeiro, M.M.A.C.; Sudo, J.T.C.; Richter, E.M.; Muñoz, R.A.A.; Silva, S.G. Talanta 2020, $217,120987$.

(4) Odoardi, S.; Romolo, F.S.; Strano-Rossi, S. Forensic Sci. Int. 2016, 265, 116-120.

(5) Fiorentin, T.R.; Logan, B.K.; Martin, D.M.; Browne, T.; Rieders, E.F. Forensic Sci. Int. 2020, 313, 110342.

(6) Parrilla, M.; Montiel, N.F.; Van Durme, F.; De Wael, K. Sens. Actuators B Chem. 2021, 337, 129819

(7) Gilbert, N.; Mewis, R.E.; Sutcliffe, O.B. Forensic Chem. 2020, 21, 100287.

(8) Jovanov, P.; Petrin-Miličević, M.; Radosavljević-Stevanović, N.; Vraneš, M.; Belić, S.; Sakač, M.; Nikolov, J.; Gadžurić, S. Anal. Lett. 2021, 54(7), 1224-1232.

(9) Angelini, D.J.; Biggs, T.D.; Prugh, A.M.; Smith, J.A.; Han-burger, J.A.; Llano, B.; Avelar, R.; Ellis, A.; Lusk, B.; Naanaa, A.M.; Sisco, E.; Sekowski, J.W. J. Forensic Sci. 2021, 66, 758-765

(10) Schram, J.; Parrilla, M.; Sleegers, N.; Samyn, N.; Bijvoets, S.M.; Heerschop, M.W.J. Anal. Chem. 2020, 92, 13485-13492.

(11) Liu, C.-M.; He, H.-Y.; Xu, L.; Hua, Z.-D. Drug Test Anal. 2021, 13, 720-728.

(12) Duarte, L.O.; Ferreira, B.; Silva, G.R.; Ipólito, A.J.; de Oli-veira, M.F. J. Liq. Chromatogr. Relat. Technol. 2021, in press. 
Table S.7 General classification for GAPI index

\begin{tabular}{cc}
\hline \multicolumn{2}{c}{ Category } \\
Green Yellow Red & Note \\
\hline
\end{tabular}

\section{Sample preparation}

Collection (1)

Preservation (2)

Transport (3)

Storage (4)

Type of method: direct or indirect (5)

Scale of extraction (6)

Solvents/reagents used (7)

Additional treatments (8)

Reagents and Solvents

Amount (9)

Health hazard (10)

Safety hazard (11)

\section{Instrumentation}

Energy (12)

Occupational hazard (13)

Waste (14)

Waste treatment (15)

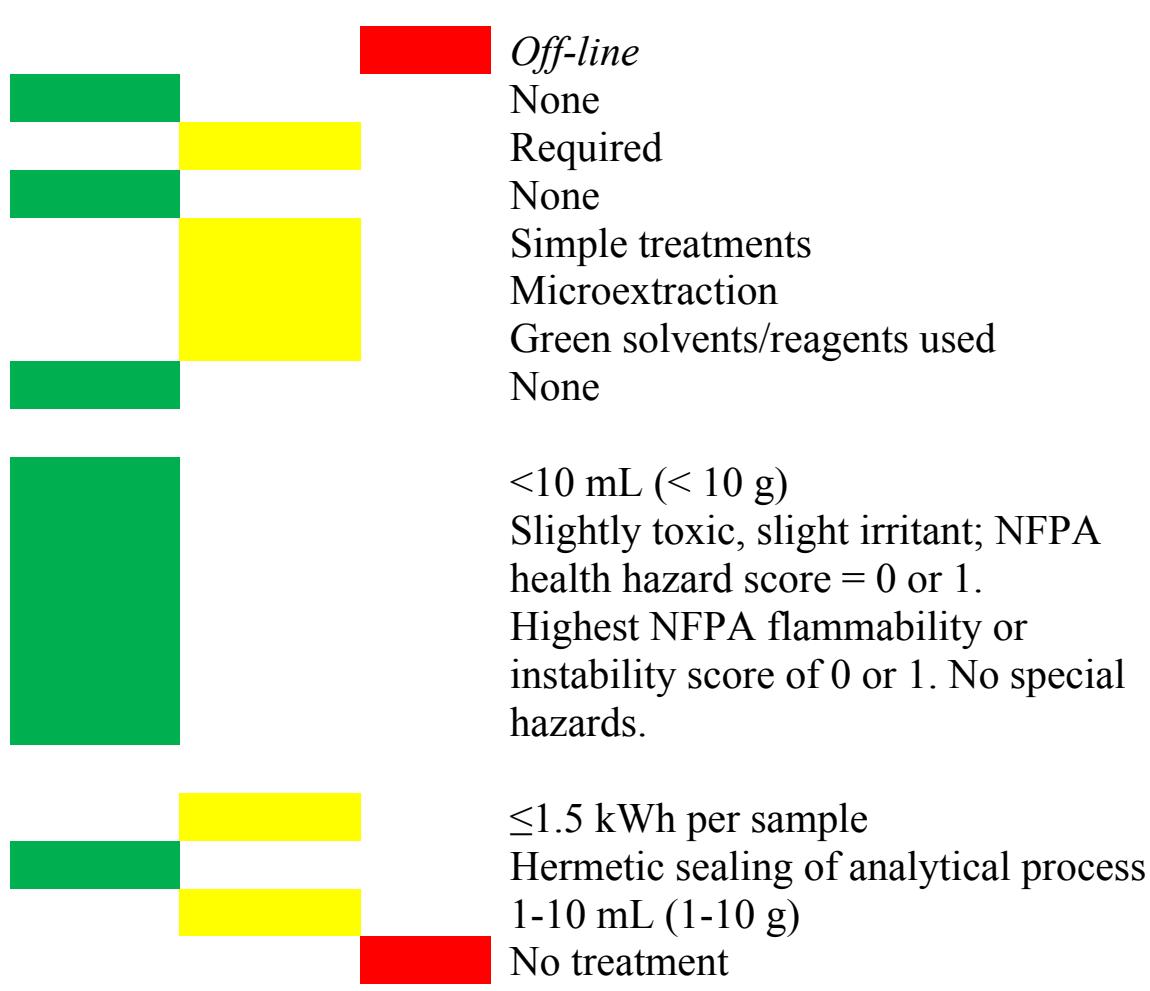

Additional Mark: Quantification

Circle in the middle of GAPI: procedure for quantitative and qualitative analysis

Figure S.4 Correspondence to GAPI pictogram in the main text:

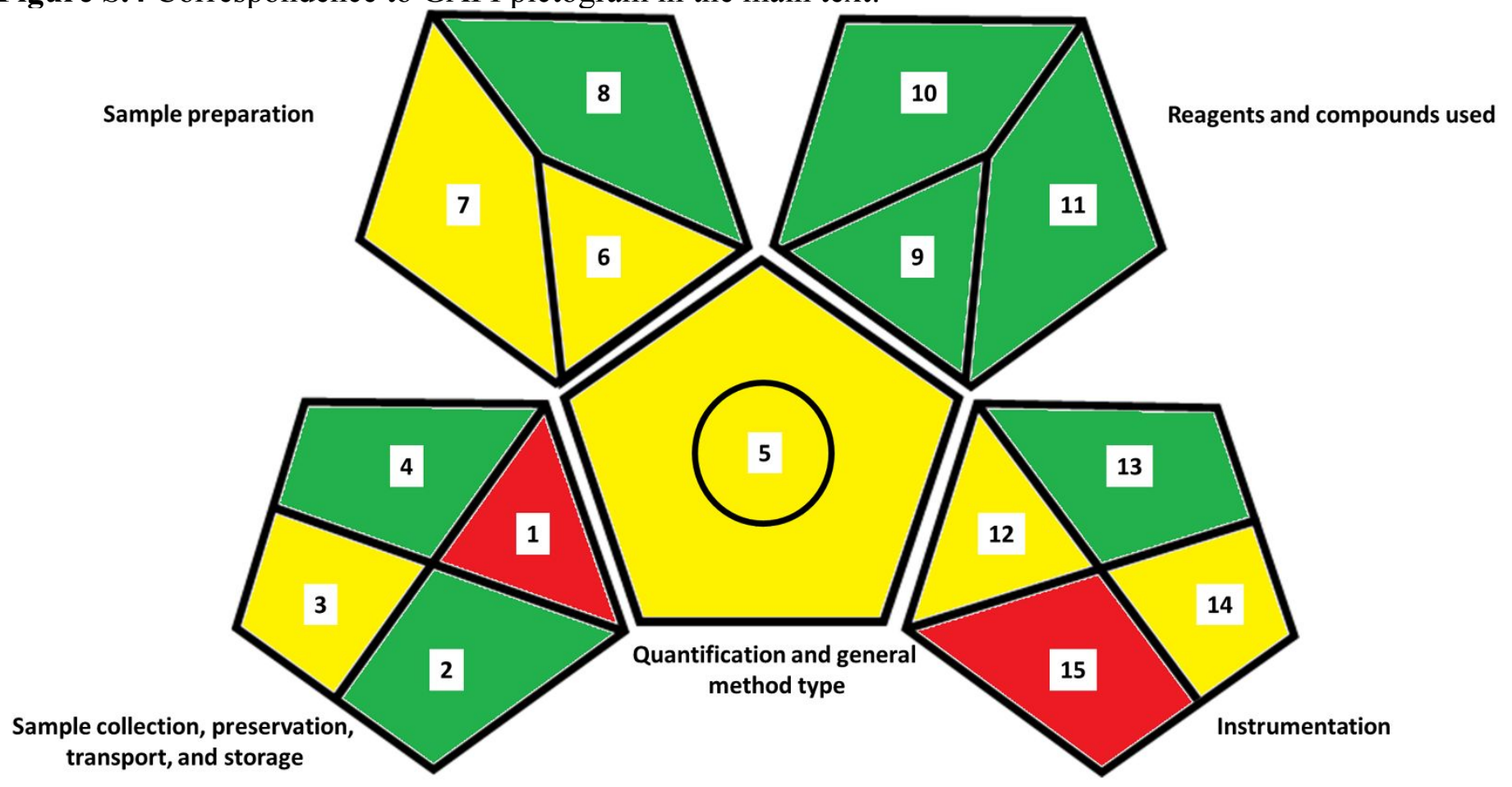

